\title{
HUMAN RESOURCE MANAGEMENT PRACTICES IN THE BOTSWANA HEALTH SECTOR
}

\author{
Theophilus TSHUKUDU*
}

\begin{abstract}
Very few studies have been done on the assessment and performance of Botswana public hospitals. Using the World Health Organization Health Systems Performance Assessment Framework (WHO HSPAF), we are going to determine the performance of health care system in Botswana. We aim to evaluate performance of Botswana public hospital system; relate findings of the assessment to the potential for improvements in hospital performance; and determine the usefulness of the WHO HSPAF in assessing performance of hospital systems in a developing country.

Keywords: Botswana public hospitals; performance of health; hospital systems
\end{abstract}

DOI: $10.53373 /$ VULS.2021.38.2.008

\section{Introduction}

Public hospitals are a central component of the Botswana health care system, accounting for over $60 \%$ of hospital beds and national health sector expenditure. These hospitals play an implacable major role in delivering preventive, curative, diagnostic, and rehabilitative services. They act as referral centers from primary healthcare facilities. The hospitals, therefore, influence performance of the entire health system. In Botswana, communities are very critical of hospitals evidenced by the negative media reports. Internationally health- policy makers and managers are trying to alternatives interventions aimed at improving performance of public hospitals. However, there is lack of systematic analysis of how hospitals function and perform, and the challenges they face, that could be used to support policy decisions and strategies for improvement.

\section{Background on ministry of health Botswana}

The ministry of health is mandated to oversee and delivery of health services for Batswana. It is responsible for the formulation of policies, regulations and guidelines for health service. The ministry is also a major provider of health services through a wide range of health facilities and management teams (DHMTs). DHMTs are responsible for running a network of health facilities, hospitals, clinics, health posts as well as community based preventatives and promotions services.

Challenges of HRM in Botswana public health care sector service include the following:

Organization and governance, Service and availability, Service capacity, service quality

\section{Organization and governance}

This addresses the organizational arrangements, leadership and management in public hospitals. Organizational arrangements have been found to have a heartfelt impact on service delivery even when the resources are available. The focus of this research is on how the

\footnotetext{
* Department of Management; Faculty of Business; University of Botswana.
} 
public hospital system is organized in relation to the (MoH), leadership structures within the hospitals, and locus of authority and decision-making.

\section{Service and availability}

This area assesses physical access to services, giving an indication of how resources are distributed across the population. Specific indicators include the proportion of the population within a specified distance of a health facility, the number and distribution of inpatient beds per 10,000 populations reflecting the ease of physical access, and access to inpatient care respectively

\section{Service capacity}

This addresses the ability of the facilities to deliver health services. It is determined by the availability of resources which include medical equipment, medical supplies, medicines, motivated stuff and hospital buildings.

\section{Service quality}

For health interventions to promote health and save lives, they must be of acceptable quality. The indicators in this area include availability of structures and strategies to improve quality of care and the perceptions of providers and users of health services about the quality of services. Botswana has a total of twenty-six public hospitals across the country. The hospital system is distributed into three levels based on the population served and the complexity of services provided. Sixteen hospitals are representing the lower level of hospital service. They are found in smaller villages which a bed capacity of twenty to seventy beds, and serves a population of ten thousand. The seven district hospitals represent the next level of care. They are found in major villages and towns with bed capacity of seventy one to two hundred and fifty beds. The three referral hospitals (two general and one psychiatric) provide specialist services in various areas. All public hospitals are directly under the $\mathrm{MoH}$ which provides policy guidance, overall supervision and all resources necessary for delivery of services. Until recently primary healthcare was the responsibility of the Ministry of Local Government.

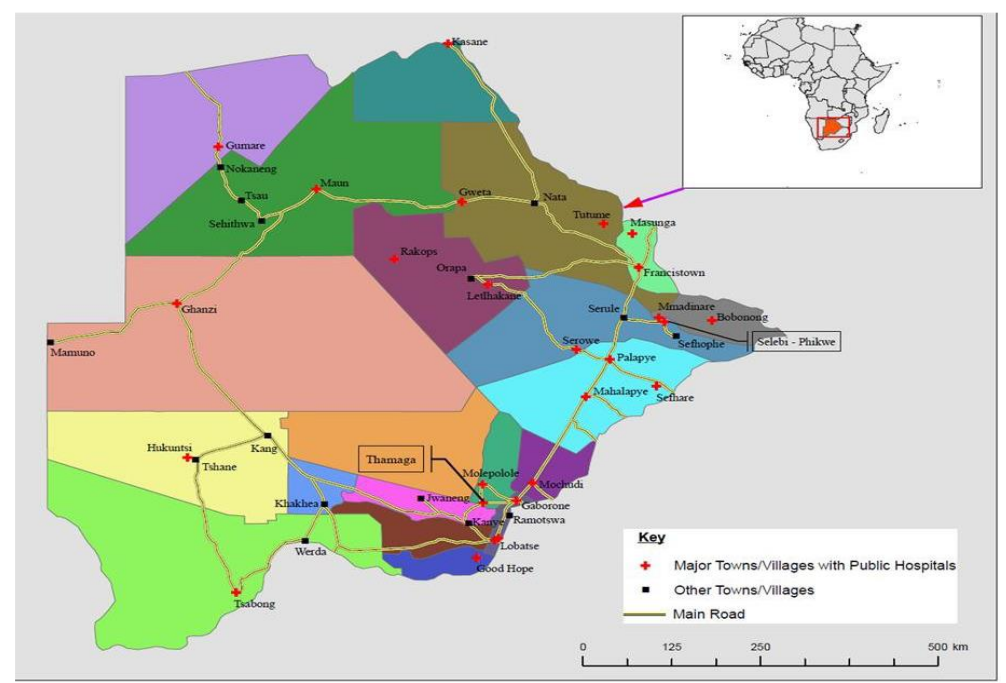

Figure 1. Map of Botswana showing public hospitals 


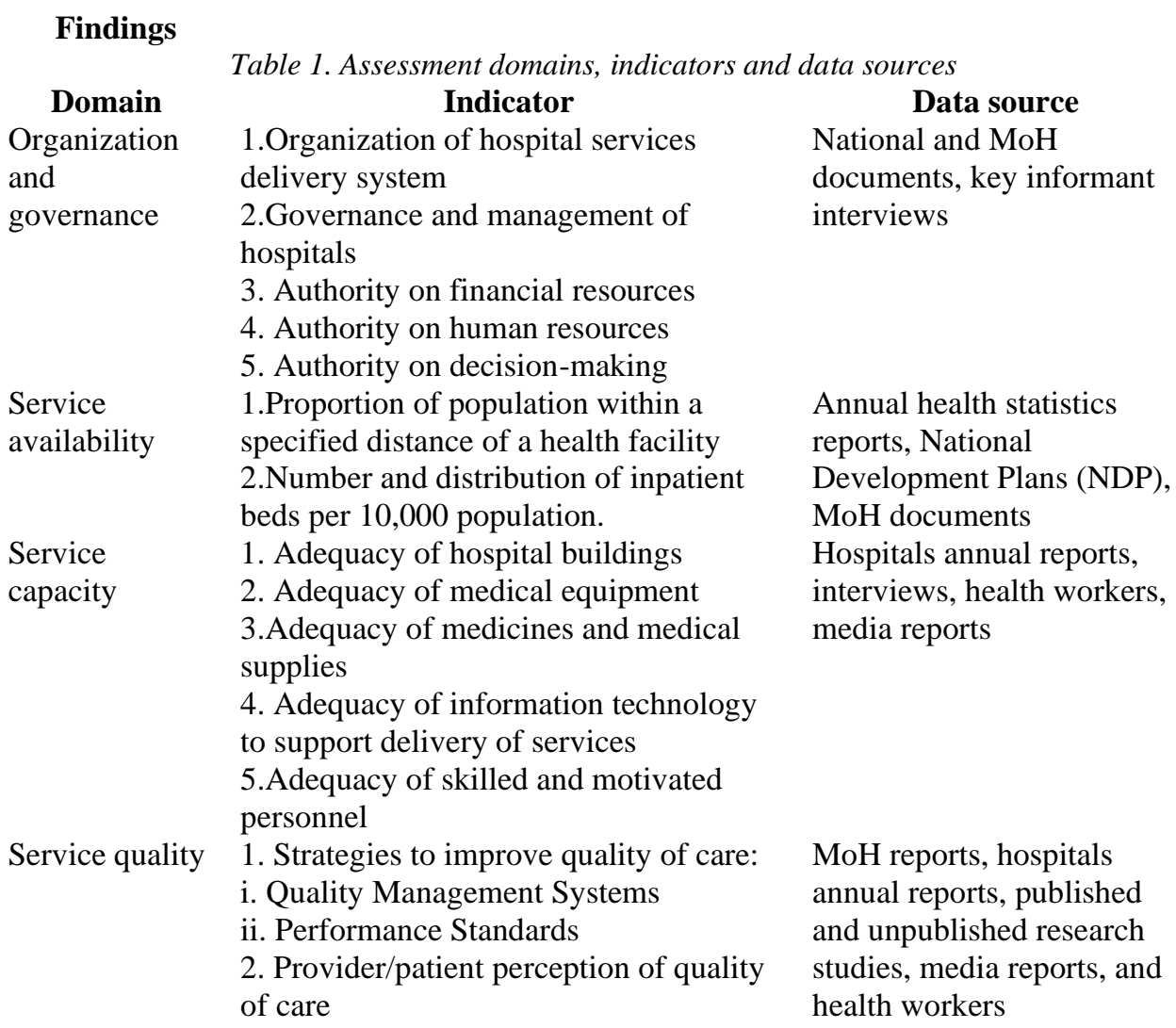

\section{Organizational structure and leadership}

All public hospitals fall under the Department of Clinical Services at the $\mathrm{MoH}$ headquarters. Hospitals are headed by Medical superintendents/Chief Medical Officers who report directly to the Director of Clinical Services, who then reports to the Deputy Permanent Secretary-Health Services who is accountable to the Permanent Secretary. This hierarchical and centralized structure thus creating challenges in management of these hospitals, for example: Since system is heavily centralized, heads of departments or hospital managers' report to the headquarters which results in no structure between the Ministry of Health and the facilities at rural areas across the country. Supervision of the head of that hospital is done centrally at the Ministry of Health, which poses the question of how reliable is the supervision if they are a thousand kilometers from where the hospital were work is done?

The senior management team for each hospital includes the hospital superintendent/chief medical officer and heads of department such as nursing superintendents or matrons and the most senior administration officer. The $\mathrm{MoH}$ created positions of hospital managers in referral hospitals and district hospitals to improve the general administration and financial management. A number of issues were brought up from this, for example: if the doctor is in charge of managing the hospital, he/she mainly focuses on the clinical service thus neglecting non- clinical aspects of the hospital such as 
administration and engineering services. Lack of clarity between the role of the hospital superintendents and hospital managers is also an issue. Educational preparation of nonclinical hospital managers is also a source of concern. Qualifications for these hospitals managers' positions are usually poorly defined and in some cases inappropriately. For example, we have hospital managers who are accountants, HR [Human Resource] professionals, economics graduates.

\section{Authority and decision-making}

The hospitals receive their budgets from the department of clinical services. Once the budget has been allocated, managers have limited authority on the use of funds. They do not have much of a decision except to follow the set rules of using the money from the votes for the purposes for which they were allocated for which comes from the ministry of health. Lack of control on human resource functions in particular recruitment and staff promotions was also a concern.

\section{Service capacity}

Status of hospital buildings

The physical infrastructure for health in Botswana has been described as more than adequate. The newly upgraded district hospitals were described as some of the best in the region. A lack of maintains was raised as a concern; hospitals and clinics in some reigns/villages are in shabby conditions as a result of this.

\section{Availability of functional medical equipment}

While most hospitals, particularly in rural areas struggled with lack of equipment, others have been equipped with modern and sophisticated machinery. Where such equipment was reported, some concerns were raised on its appropriateness and the ability to optimize its use considering the skills and experiences of personnel.

\section{Service quality}

Quality management systems

According to Seitio-Kgokgwe et al, quality improvement strategies and plans were observed to be outdated in 2009. Hospitals were also seen to lack focus on quality issues characterized by lack of evidence of clinical governance structures and strategies, failure to address quality issues in management meetings, and lack of procedures and evidence of conducting clinical audits or monitoring effectiveness of car.

\section{Availability of skilled and motivated human resources}

Hospitals reported a general shortage of staff that limits delivery of services and achievement of planned hospital activities. The main challenges reported were in the area of specialist personnel resulting in unnecessary referrals. Challenges were cited within the human resource management processes. Lack of integrated planning characterized by disjoint in human resource, infrastructure and service delivery planning created inefficiency in public hospitals.

Availability of information technology to support delivery of health services

The MoH promoted the use of Information and Communications Technology (ICT) in our healthcare system. A number of patient management information systems were 
introduced in hospitals. These included the Integrated Patient Management System implemented in few hospitals with plans to roll it out to all facilities, tele-radiology in national referral hospitals, the Patient Information Management System dedicated to management of patients on antiretroviral treatment and cancer and Tuberculosis (TB) registration systems. While the Ministry's efforts are recognized, challenges of multiple standalone systems with limited interoperability were noted. Despite the introduction of these systems in public hospitals, health workers feel like their hospitals did not have adequate Information Technology (IT) to support provision of quality patient care.

\section{Performance standards}

A lack of national performance standards in public hospitals contributes to variability in performance. This was noted as a concern particularly considering that the majority of the medical doctors in Botswana come from different countries with different backgrounds. There are very few documented standards within the health system and those that do exist, most of the personnel are not aware of them. Basically most people work according to the standards by which they were trained for which may be good but they come from different places and they have different standards. Hospitals weak legislation and policies regulating quality of care were some of the challenges.

\section{Provider perception of the quality of care}

Healthcare providers seem to have a more positive view of the quality of services provided in public facilities. In a study conducted by the $\mathrm{MoH}$, half of the participants rated the quality of care as good. The same study cited a lack of basic equipment, an unclean environment, lack of privacy and poor referral systems as issues affecting delivery of services, despite challenges noted on availability of resources in the current study, most of the managers felt that the quality of services has improved. This view was not shared by participants from the interviews, who generally felt that there was a major quality issues in hospitals. These varied from inadequate resources from the $\mathrm{MoH}$ to support the hospitals in managing their quality related problems.

\section{Patient perceptions of quality on care}

Over the years, research conducted on patient satisfaction highlighted a myriad of concerns including inadequate staff, and negative attitudes from staff members. There are concerns about the shortage of resources, particularly health personnel, which contributes to long waiting time and poor access to services as a radio caller noted:

"Our hospitals do not have resources and there is shortage of medical doctors. It is possible to be booked to see a doctor after three months, when you come you are told he/she is not available because he is alone... You find one doctor helping so many patients, or you are told he/she is still help other patients while your own illness progresses and finally you give up and go home without assistance".

\section{Service availability}

Botswana has invested enormously in health infrastructure in terms of hospitals and primary healthcare clinics ensuring access to services. There are considerable challenges facing tertiary care in the Botswana's two national referral hospitals (Princess Marina Hospital and Nyangagwe Referral Hospital). The lack of lower level hospitals where these referrals hospitals are located has a profound impact on their functioning. These challenges 
may include limited access to tertiary care for populations outside the two cities as the referral hospitals are forced to function as local hospitals.

\section{Service capacity}

Although it was not possible to accurately determine the number of health workers in Botswana's public hospitals due to lack of a robust human resource information system, hospitals throughout the country reported inadequate numbers particularly in specialist areas. While health worker shortages are a global problem especially in developing countries, the critical issue for Botswana is effective utilization of human resources. Poor deployment creates inefficiencies through an inappropriate skill match which may contribute to poor service quality and staff motivation. Poor staff retention is also a concern that contributes to high attrition rates. Inadequate maintenance of hospital buildings, non-functional and inequitable distribution of equipment, and poor availability of medicines are some of the key challenges facing public hospitals in Botswana. These challenges will need to be addressed if acceptable levels of healthcare services are to be provided.

\section{Service quality}

Public hospitals are faced with a myriad of quality related problems. The absence of quality management and improvement structures, and lack of practice standards to guide delivery of services had a negative impact on the quality of care. According Seitio-Kgokgwe et al, Several participants from the MoH cited the proposed establishment of the Health Inspectorate department in the $\mathrm{MoH}$ as an opportunity to improve the quality of patient care in public hospitals. As a result, $\mathrm{MoH}$ has been in talks with the Council for Health Services Accreditation of Southern Africa (COHSASA) which is a regional organization that confers accreditation on health facilities that comply with specified quality standards. Accreditation is reported to have been piloted in a few hospitals. The success of these initiatives, however, will depend on the nature and strength of incentives to motivate behavior change within the hospitals. Accountability is identified as one of the elements that influence hospital performance. Strong accountability mechanisms will be needed to hold managers responsible and accountable for good results.

Usefulness of the World Health Organization Health System Performance Assessment Framework in assessing performance of hospitals

We used an aspect of the WHO HSPAF, the health service delivery function, to guide assessment of Botswana's public hospital system.

Unlike earlier studies conducted in Botswana assessing hospital performance using economic, the use of the WHO HSPAF in this analysis provides an opportunity to take a comprehensive view of the hospital system to identify the strengths and weakness in various facets of hospital system performance. This approach is in line with the notion that health systems are complex entities that require a multidimensional approach to performance assessment. Development of performance indicators to measure performance was a key performance this. While some work has been done in this area by organizations such as USAID and WHO, the focus was on the development of indicators that will be relevant to Botswana and that would provide policy-and decision-makers with information that addressed their needs, thus improving the system.

The opportunity provided by the use of WHO HSPAF in terms of multiple assessment challenges and indicators allowed the different stakeholders which included policy-makers from the $\mathrm{MoH}$, representatives of non-governmental organizations, hospital managers, health 
workers and the general public to contribute their own view point to the analysis. In taking this multi-constituency approach, divergent views in assessment of performance were acknowledged as differing perspectives of stakeholders. The essence of measuring performance is to be able to make improvements in areas where performance is lacking. By focusing on processes, structures and resource inputs, the use of the HSPAF enabled the assessment of the hospital system to specifically indicate problems contributing to poor performance. The indicators used in this analysis are mainly process indicators as opposed to outcomes indicators. While outcome measures are useful in identifying major areas of concern, they can fail to identify levers of system performance. On the other hand, process indicators have been credited for providing feedback that can be used to improve system performance by identifying dysfunctional processes.

\section{Conclusion}

We successfully used the WHO HSPAF to conduct a comprehensive assessment of the public hospital system in Botswana. Although these hospitals are under pressure to improve performance, they are faced with considerable challenges that will need to be addressed if changes in performance are to occur. The organizational structure and governance of public hospitals have a negative impact on the hospitals' performance. Lack of management autonomy limits the ability of hospital managers to influence performance of their facilities. The ongoing efforts to establish district health management structures to coordinate and manage health service delivery at district levels will need to be expedited and sufficient capacity built to ensure that these structures can effectively handle their responsibilities. The important role of health system resources in service delivery needs to be appreciated and concerted efforts made to improve resource allocation and utilization.

\section{References:}

1. Mothibi M. Ke sechaba se dire eng gore ditlamelo di se tswele mosala. Gaborone: Radio Botswana; 2009.

2. Mothibi M. A batho ga ba tshephe bongaka jwa mo gae. Gaborone: Radio Botswana; 2010 .

3. Murray CJL, Frenk J. A framework for assessing the performance of health systems. Bull World Health Organ 2000; 78: 717-31.

4. Ramanathan TV, Chandra KS, Thupeng WM. A comparison of the technical efficiencies of health districts and hospitals in Botswana. Dev South Afr 2003; 20: 307-20.

5. Seitio-Kgokgwe O. Organizational Structure of the Botswana Ministry of Health: Impact on Performance. Dunedin: University of Otago; 2012.

6. Tlotlego N, Nonvignon J, Sambo LG, Asbu EZ, Kirigia JM. Assessment of productivity of hospitals in Botswana: A DEA application. Int Arch Med 2010; 3: 27. doi: 10.1186/1755-7682- 3-27.

7. ***Central Statistics Office (CSO). Health facility by type and number of beds $1998-2006$, 2009.

8. ***Ministry of Health (MoH). Republic of Botswana National Health Accounts, 2006. Gaborone: Ministry of Health; 2006. 